\title{
La gouvernance des trames vertes et bleues : de la nécessité d'une approche opérationnelle et réflexive
}

\author{
Valérie ANGEON ${ }^{1}$ \\ Arnaud LARADE ${ }^{2}$
}

\section{Résumé}

La gouvernance des trames vertes et bleues, nouveaux instruments de conservation de la biodiversité, invite à de nombreuses innovations qui réclament des apprentissages collectifs. De ce point de vue, le dispositif ALDOM, testé en Guadeloupe, est concluant sous réserve d'y adjoindre la dimension stratégique des rapports interindividuels peu explorée dans la littérature sur la Gouvernance Adaptative.

\begin{abstract}
The governance of green and blue infrastructures (tool for biodiversity conservation) calls for various innovations that require collective learning mechanisms. Tested in Guadeloupe, the implementation of the method ALDOM allows consistent results as long as strategic games and power relationships are taken into account. Adaptive governance consists in bringing in these results for further actions.
\end{abstract}

\section{Introduction}

Issues du Grenelle de l'environnement, les trames vertes et bleues (TVB) sont dédiées à la conservation de la biodiversité en France. Elles ont pour objectif de permettre les capacités de libre évolution du vivant par le maintien ou la

1 Valérie ANGEON : Directrice de recherche, INRA, Unité de Recherches Zootechniques UR 143 - valerie.angeon@inra.fr

2 Arnaud LARADE : Ingénieur de recherche, AgroParisTech - alarade971@yahoo.fr 
création de continuités écologiques. Elles sont structurées autour de réservoirs de biodiversité (forêts, dunes, zones humides, cours d'eau, etc.) reliés par des corridors écologiques (haies, ripisylves, bosquets, mares, etc.) dont les tailles et les formes varient.

Les TVB doivent être traduites dans différents dispositifs de planification et d'aménagement du territoire qui doivent être compatibles. Leur conception et leur mise en œuvre imposent le principe d'une " gouvernance à cinq ». Il s'agit d'intégrer dans les processus de décision et d'action cinq collèges d'acteurs représentant les collectivités territoriales et leur groupement, l'État et ses établissements publics, les organisations professionnelles et les usagers de la nature, les gestionnaires des espaces naturels, les scientifiques et personnalités qualifiées.

Ainsi présentées, les TVB sont des systèmes écologiques et sociaux qui doivent concilier les activités humaines et la mobilité du vivant. Toutefois, les interactions entre les dynamiques écosystémiques et les logiques socio-économiques dans lesquelles s'inscrivent ces systèmes, les limites des connaissances scientifiques et profanes disponibles, l'imbrication des niveaux et des échelles de décision, la diversité des intérêts des parties prenantes qui interviennent dans la gestion de ces systèmes créent des configurations inédites de complexité et d'incertitude.

Ce contexte de complexité et d'incertitude invite à des innovations en matière de gouvernance afin de définir et de légitimer socialement les objectifs et les normes en matière de conservation de la biodiversité (Vimal et al., 2012 ; Charvolin et al., 2011 ; Vimal et Mathevet, 2011) et de faciliter la prise de décision et d'action communes. Ces innovations doivent également favoriser les processus d'ajustement continus des systèmes écologiques et sociaux considérés et les apprentissages qui y sont liés. Cette problématique est au cœur du programme de recherche de la gouvernance adaptative (Folke et al., 2005 ; Termeer et al., 2010 ; Chaffin et al., 2014). Néanmoins, ce champ de littérature reste peu précis sur les méthodes, les procédures, les outils et les instruments à mettre en œuvre pour assurer la gestion effective des systèmes considérés.

Cet article propose un outil opérationnel de gouvernance adaptative (GA) testé dans le cadre de la mise en œuvre des TVB en Guadeloupe. Il s'agit du dispositif participatif ALDOM (Association, Language, Diagram block, Original Method) qui vise à favoriser l'hybridation des connaissances, le raisonnement systémique et les dynamiques d'apprentissage collectif nécessaires à la définition et à la mise en œuvre des TVB. Les résultats de l'application de ce dispositif nous permettent de discuter du caractère opératoire de la démarche proposée. Ce regard critique sur l'outil et son implémentation autorise une réflexion d'ordre général sur l'importance de la dimension stratégique dans les processus de GA.

Trois temps forts structurent ce texte. Premièrement, après avoir exposé les fondements de la GA, nous élaborons une proposition méthodologique pour en opérationnaliser le contenu. Deuxièmement, nous présentons le disposi- 
tif ALDOM conçu et implémenté pour répondre aux objectifs d'innovation et d'apprentissage que réclame la mise en œuvre des TVB. Enfin, nous exposons et discutons les résultats d'une application de ce dispositif à Morne-à-l'Eau en Guadeloupe, seule commune de l'archipel à avoir déposé et obtenu du ministère en charge de l'Écologie le financement d'un projet de restauration des TVB.

\section{La gouvernance adaptative : une théorie métaphysique de l'action collective}

En tant que cadre d'analyse à visée opérationnelle (Folke et al., 2005 ; Termeer et al., 2010), la GA se présente comme une théorie normative pour la gestion des systèmes écologiques et sociaux (Chaffin et al., 2014). La GA fait explicitement de la capacité des acteurs à organiser et à inclure le retour d'expériences dans les processus de gestion des systèmes écologiques et sociaux une condition de réussite de leur préservation effective. Elle place ainsi au cœur de l'action publique la question de la reconnaissance et de la légitimité des acteurs impliqués dans la gestion des systèmes écologiques et sociaux dont les représentations en matière de biodiversité peuvent différer et dont l'état des connaissances sur les objets d'environnement est plus ou moins complet. Ce faisant, la GA invite à raisonner sur le rôle des espaces institutionnels et des dispositifs socio-techniques facilitateurs des transactions sociales nécessaires entre les différentes catégories d'acteurs pour permettre un travail de production cognitive et d'action collective. La GA s'inscrit en cela en filiation aux travaux sur la gouvernance (1.1) en même temps qu'elle procède à son enrichissement en invitant à entreprendre une réflexion méthodologique pour opérationnaliser le concept (1.2).

\subsection{Qu'est-ce que la gouvernance adaptative?}

La GA a d'abord été développée comme une approche de gestion et a ensuite évolué en un champ interdisciplinaire de recherche et d'action sous le terme générique de "gouvernance adaptative » (Folke et al., 2005 ; Termeer et al., 2010). La GA a émergé dans les années 1970 comme une réponse scientifique avec pour objectif opérationnel d'éclairer les modalités de gestion des systèmes écologiques et sociaux caractérisés par la complexité et l'incertitude (Berkes et Folke, 1998 ; Gunderson, 1999 ; Dietz et al., 2003 ; Walker et al., 2004 ; Anderies et al., 2004). La revue de littérature établie par Chaffin et al. (2014) la présente comme une approche générale de gouvernance de l'environnement. En cela, la GA analyse les relations entre entités vivantes humaines au sujet de l'existence (ou non) d'entités vivantes non humaines. Elle s'intéresse aux processus de régulation, aux mécanismes et aux organisations par lesquels les acteurs prennent part à l'action (Lemos et Agrawal, 2006).

Partant du principe élémentaire que tout système écologique (qu'il soit anthropisé ou non) s'insère dans un cadre sociétal, la GA considère que le contexte 
sociétal (qualité du rapport homme-nature, systèmes de normes et de valeurs, règles édictées, etc.) influe sur le système écologique. Est alors explicité comment une matrice institutionnelle et sociale (à travers notamment l'état de la coordination locale) peut préserver (ou non) les systèmes écologiques et sociaux (Angeon et Caron, 2009). Cette littérature invite à mettre l'accent sur les règles de décision que prennent les agents pour gérer les systèmes écologiques et sociaux. Elle s'inscrit dans une lecture critique du rôle descendant et unilatéral de l'État dans la gestion de l'environnement au moyen d'instruments réglementaires de type contrôle-commande (command and control) dont l'efficacité économique et environnementale est discutée au profit d'une approche ascendante portée par des acteurs locaux s'engageant collectivement dans la définition des enjeux, des objectifs et des normes de préservation de l'environnement. Si ces modalités de gouvernance par les acteurs locaux se sont progressivement imposées à la fois dans la littérature et comme cadre d'action pragmatique, elles font aussi l'objet de critiques (Cosens et al., 2014). Leur efficacité en matière d'environnement reste contingente aux acteurs qui prennent part au processus de décision. Des acteurs peuvent être exclus de l'arène de décision. Dès lors, certaines connaissances, pourtant essentielles à la compréhension des systèmes écologiques, peuvent être ignorées.

Bien qu'elle formalise un cadre d'action pragmatique, dans la pratique, nombre d'acteurs gestionnaires des milieux naturels (anthropisés ou non) méconnaissent les principes de la GA. Ces acteurs ont en effet souvent le sentiment de pratiquer spontanément une gestion adaptative des écosystèmes dont ils ont la charge, soit - comme l'indiquent Cordonnier et Gosselin (2009) à propos des écosystèmes forestiers - que leurs pratiques de gestion : 1) font consensus (elles sont en cela ratifiées et éprouvées au cours du temps et par conséquent de facto adaptées) ; 2) s'ajustent aux divers contextes socio-politiques et écologiques dans lesquels elles s'appliquent (elles sont évolutives) ; 3) favorisent l'évolution du milieu et conséquemment son adaptation aux changements ou chocs externes ou internes. Dans ces trois configurations, le caractère adaptatif de la gestion est spontané et systématique. Il n'y a pas de remise en cause des pratiques adoptées.

Or, ce qu'on appelle gestion adaptative consiste à « réaliser des retours d'expérience structurés au sujet des modes de gestion pratiquée afin d'approfondir nos connaissances sur la relation milieu-gestion. Pour qu'une gestion soit considérée comme adaptative, il faut que l'acquisition de connaissances ou d'expériences soit délibérément organisée au sein du processus de gestion lui-même et qu'ily ait une procédure définie pour adapter les pratiques futures en fonction de cet apprentissage » (Cordonnier et Gosselin, 2009, p. 3). En d'autres termes, on entend par une gestion effectivement adaptative une activité de gestion qui repose sur les apprentissages des politiques et pratiques passées et influence réellement les stratégies de gestion à mettre en œuvre dans le futur pour initier le changement (Folke et al., 2005 ; Pahl-Wostl et al., 2007 ; Termeer et al., 2010). 
La GA met l'accent sur les compromis environnementaux et la définition normative des enjeux et des objectifs de préservation de l'environnement. Elle conçoit que le travail de production de ces normes d'action et de leur appropriation ne peut pas s'effectuer par le biais d'un simple transfert de nouveaux référentiels techniques issus des champs scientifiques et techniques (Theau et al., 2012). La co-production partenariale de ces normes d'action en associant la communauté scientifique et les gestionnaires des espaces apparaît indispensable. La GA mobilise pour cela divers apports disciplinaires (sciences exactes, sciences du vivant, sciences de la société) et convoque différentes formes de connaissances (scientifiques ou profanes) et de savoirs.

Compte tenu de la complexité et de l'imprédictibilité des interactions entre les systèmes écologiques et sociaux, du caractère lacunaire et non stabilisé des connaissances disponibles, des controverses liées à l'efficacité des dispositifs d'action publique pour la préservation effective des systèmes considérés, les modalités de l'accompagnement au changement doivent être interrogées. Elles doivent être envisagées dans une perspective d'amélioration incrémentale continue.

Aussi, nous retenons que raisonner dans le cadre théorique de la GA revient à (1) concevoir des activités de gestion (2) tout en intervenant sur les systèmes (socio-écologiques) par la mise en œuvre effective d'activités de gestion. Le programme de recherche de la GA doit dès lors s'attacher à produire et à utiliser de manière systématique une méthode opérationnelle. Il s'agit en effet d'éviter de confondre le processus à suivre pour rendre une activité de gestion effectivement adaptative et la GA en tant que réalité achevée et de ne pas laisser apparaître la GA comme une théorie métaphysique de l'action collective (Hatchuel, 2012).

\subsection{Opérationnaliser la gouvernance adaptative}

Cadre d'analyse pertinent pour rendre compte des capacités de réponse des systèmes écologiques et sociaux en situation d'incertitude, la GA cherche à se doter d'un véritable agenda de recherche pour rendre possible l'action et l'améliorer chemin faisant (Sainsbury et al., 2000 ; Smith et al., 1999; Dutra et al., 2015). Opérationnaliser la GA c'est, d'une part, expérimenter l'action sur le terrain et, d'autre part, effectuer un retour réflexif à partir de cette expérience pour enrichir la théorie. La GA serait bien ainsi une approche théorique qui se nourrit de l'action.

En nous saisissant de l'exemple des TVB, nous montrons comment les principales conditions de leur élaboration fixées par la loi (prise en compte de la biodiversité ordinaire, articulation d'échelles spatiales, participation) interrogent la capacité à rassembler les acteurs, les modalités d'expression et de mobilisation de leurs connaissances, la mise en comptabilité de leurs représentations et de leurs intérêts. Nous proposons sur cette base une démarche pour faciliter le cadre de l'action. 
Avec les TVB, la nature « sort de sa réserve », montrant l'importance des espaces dits « ordinaires » dans la conservation de la biodiversité au même titre que les espaces à haute valeur naturelle. Les TVB impliquent ainsi de revaloriser la place des entités vivantes non humaines au sein des collectifs humains.

Les processus de définition des TVB doivent mettre en relation des territoires qui ne l'étaient pas forcément auparavant. En effet, les TVB ne se déclinent pas nécessairement sur des espaces où existaient déjà des solidarités territoriales et des coopérations. L'institutionnalisation des continuités écologiques via les TVB entraîne non seulement une recomposition des espaces et des échelles, mais provoque aussi de nouvelles interdépendances susceptibles de faire émerger des espaces de décision inédits.

Formellement, les orientations nationales en matière de TVB doivent être prises en compte à l'échelle régionale (par les Schémas Régionaux de Cohérence Écologique, SRCE), eux-mêmes pris en compte dans les documents d'aménagement et de planification des collectivités territoriales (aux niveaux communal et intercommunal), en particulier les documents d'urbanisme (Plans Locaux d'Urbanisme, PLU, et Schémas de Cohérence Territoriale, SCoT). Les milieux et les écosystèmes doivent être conçus de manière continue ou liée entre eux. Formant ainsi des « objets chevelus » (Latour, 1995), la compréhension de leur principe de fonctionnement et leurs modalités de gestion nécessitent que les parties prenantes fassent l'apprentissage d'un raisonnement spatial réticulaire (Alphandéry et Fortier, 2012) qui implique des considérants de long terme. Autrement dit, les acteurs ne doivent plus considérer leurs actions simplement « ici et maintenant » mais « ici et là-bas, maintenant et demain ».

Avec l'introduction du principe de la " gouvernance à cinq », la conception et la mise en œuvre des TVB constituent un travail politique de réassemblage qui nécessite de coordonner ces acteurs jusqu'alors absents des instances de délibération. L'un des défis des TVB repose ainsi sur la coordination d'acteurs qui ont des visions, des représentations, des pratiques - i.e. sectorielles (agriculture, transports, développement économique, protection des milieux naturels...) -, des normes d'action, des usages différenciés de l'espace pour arriver à une co-élaboration et à un partage de référents sur ce que doivent être les continuités écologiques sur leur territoire. Est bien ici en jeu la question de l'articulation entre les dimensions individuelles et collectives des représentations, des normes d'action, des mécanismes d'apprentissage. Cette mise en compatibilité des référentiels individuels et collectifs est un élément important pour la mise en œuvre et la gestion effective des TVB. Il convient donc de réfléchir à la méthode de gestion susceptible de faciliter l'opérationnalisation du dialogue territorial. Nous proposons pour cela une démarche méthodologique qui comporte quatre phases essentielles (Figure 1).

La première consiste à rassembler les parties prenantes, ce qui suppose de les identifier et de les mobiliser. Un principe de reconnaissance et de légitimité de ces acteurs porteurs de savoirs et de référentiels diversifiés doit être observé. 

approche opérationnelle et réflexive

La deuxième phase a pour objectif de faire exprimer aux acteurs impliqués leurs représentations et leurs connaissances des enjeux d'environnement. Elle doit déboucher sur une mise en commun des savoirs et la définition d'une vision partagée des entités de biodiversité à préserver. La troisième phase vise à définir les priorités, les moyens et la programmation des actions. Enfin, la quatrième phase permet de définir et d'évaluer les apprentissages acquis. Pour être transformant, les apprentissages doivent modifier les systèmes de valeurs des acteurs. Cela renvoie au processus par lequel les individus modifient leurs croyances, points de vue, représentations, schèmes antérieurs sous l'effet des nouvelles perspectives tirées de leurs apprentissages. Les résultats acquis constitueront un socle de savoirs et de sens communs qui, dans une prise de recul critique, viendront enrichir l'expérience collective de gouvernance (Dietz et al., 2003 ; Folke et al., 2005 ; Olsson et al., 2006 ; Pahl-Wostl, 2009 ; Termeer et al., 2010).

\section{Figure 1 - Un processus collectif d'expérimentation de l'action}

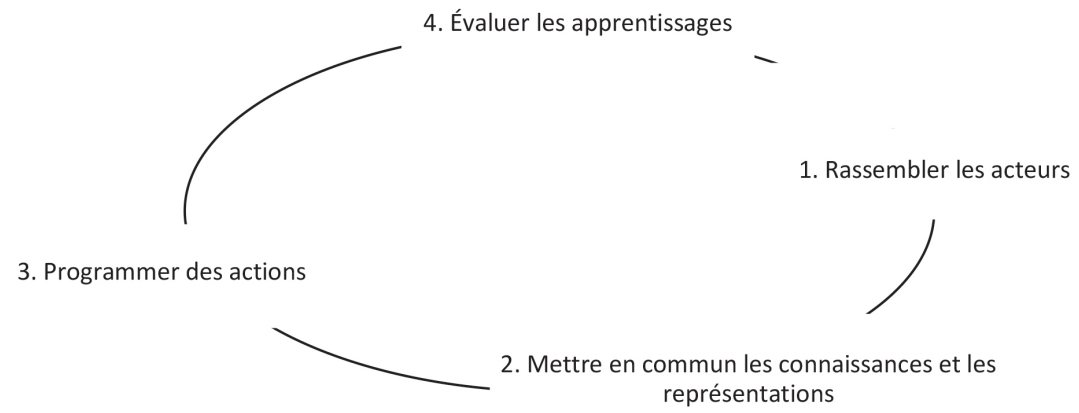

À partir des apprentissages réalisés par les acteurs, il est possible d'opérer une lecture compréhensive du processus de gouvernance qui est engagé. Cela permet d'identifier les éléments susceptibles d'enrichir la GA. En effet, dans une perspective réflexive, ces éléments devront être réintégrés dans la suite du processus de gouvernance pour promouvoir le changement de manière effective et rendre la dynamique engagée véritablement adaptative. C'est bien l'intégration de ce retour d'expérience qui est central dans la GA. La GA est en cela le résultat d'un processus cumulatif d'essais et d'erreurs.

Plus largement, notre proposition méthodologique participe à l'enrichissement de la GA en conceptualisant le statut de l'action dans une posture de recherche compréhensive. Cette posture implique d'opérer des allers-retours entre le matériau empirique et le cadre théorique. Opérationnaliser la GA, c'est intégrer les résultats du terrain dans la construction de la théorie. Si les résultats de l'action permettent de dégager des grilles de lecture, d'interprétation et de 
compréhension du réel, cette lecture compréhensive permet quant à elle le travail de distanciation critique par rapport à l'action et en améliore le pilotage. Action et compréhension s'incrémentent l'une l'autre (Figure 2).

\section{Figure 2 - Opérationnaliser la gouvernance adaptative : quand l'action et la compréhension s'incrémentent}

\section{Expérimenter l'action}

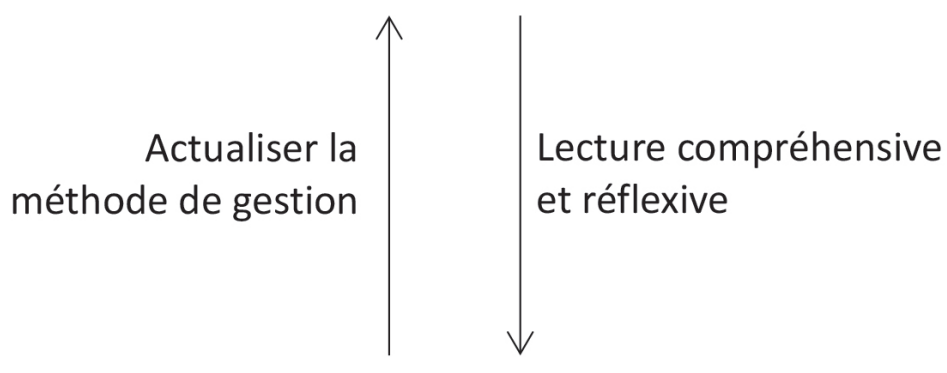

Enrichir la théorie de la gouvernance

Opérationnaliser la GA, c'est conduire une démarche de facilitation de l'expérimentation de l'action et de réflexivité. Dans les propos qui suivent, nous illustrons cette proposition méthodologique.

\section{Mettre en œuvre la gouvernance adaptative. L'exemple de la TVB en Guadeloupe}

La gouvernance adaptative des TVB réclame non pas de «transmettre des connaissances et des recommandations » aux acteurs, mais de les faire participer à « l'exploration et la transformation de la problématique » (Charvolin et al., 2011, p. 75) posée par la définition et la mise en œuvre des TVB sur leur territoire. L'opérationnalisation d'un tel processus de gouvernance rend nécessaire le développement d'outils. Dans cette section, nous proposons un dispositif socio-technique qui vise à systématiser l'expérimentation de l'action (2.1). Il s'agit d'un dispositif participatif qui favorise des apprentissages pour promouvoir le changement. Il a été testé en Guadeloupe dans la commune de Morne-à-l'Eau en milieu agricole (2.2). Les résultats de cette expérience autorisent une montée en généralité sur le concept de gouvernance adaptative (2.3). 


\subsection{Un dispositif participatif d'apprentissage pour une gouvernance effective des TVB}

Afin de rendre effective la gouvernance des TVB, le dispositif participatif d'apprentissage ALDOM a été conçu. Ce dispositif vise à hybrider des régimes de production de connaissances, faciliter le raisonnement spatial et favoriser des dynamiques d'apprentissage nécessaires.

Le dispositif ALDOM a pour objectif de faire collectivement identifier et de matérialiser dans l'espace et dans un temps contraint les TVB par des non-spécialistes. Il comporte plusieurs étapes (Encadré 1) qui amènent les acteurs à définir les enjeux écologiques du territoire, les menaces qui pèsent sur ces enjeux, les pratiques de gestion ainsi que les règlements locaux permettant de préserver les entités menacées. Ces différentes étapes correspondent aux trois premiers temps forts de la démarche méthodologique présentée antérieurement (Figure 1) pour faciliter le processus collectif d'expérimentation de l'action, à savoir : rassembler les parties prenantes, mettre en commun les représentations et programmer des actions de gestion effective.

Le dispositif ALDOM mobilise quatre outils élaborés sur la base de connaissances en écologie et en géographie du paysage (Planchat-Héry, 2011) : (1) l'utilisation de mots-clés (association continuée) pour exprimer des éléments de biodiversité ; (2) des photographies contextualisées d'éléments de biodiversité ; (3) des blocs diagramme paysagers ${ }^{3}$; et enfin (4) des logotypes représentant des menaces pour les espèces et les espaces de nature (fragmentation, pollution ponctuelle, pollution diffuse, etc.). Les outils d'élicitation sont couramment utilisés dans les procédures de GA (Dutra et al., 2015). Les associations continuées et le photolangage en sont illustratifs. Par ailleurs, la mise en œuvre des TVB exige des productions cartographiques qui demeurent souvent le fait de spécialistes, ce qui ne facilite pas leur appropriation par les usagers de la société civile et les élus (Planchat-Héry, 2011 ; Bousquet, 2016). L'utilisation de blocs diagrammes paysagers et de logotypes permet de cartographier les enjeux de continuités écologiques tout en mutualisant les sources de connaissances et en favorisant leur appropriation collective. Ces quatre types d'outils permettent à chaque participant de se figurer des représentations relatives aux éléments de biodiversité en les partageant avec d'autres.

3 Le bloc diagramme paysager est un ensemble d'éléments paysagers synthétisant les caractéristiques spécifiques du territoire sur lequel les acteurs doivent raisonner. Tel qu'il se présente, c'est certes un territoire fictif que chacun s'approprie aisément parce qu'il concentre des objets spatiaux structurants à partir desquels les acteurs se projettent. 


\section{Encadré 1 - Le dispositif ALDOM}

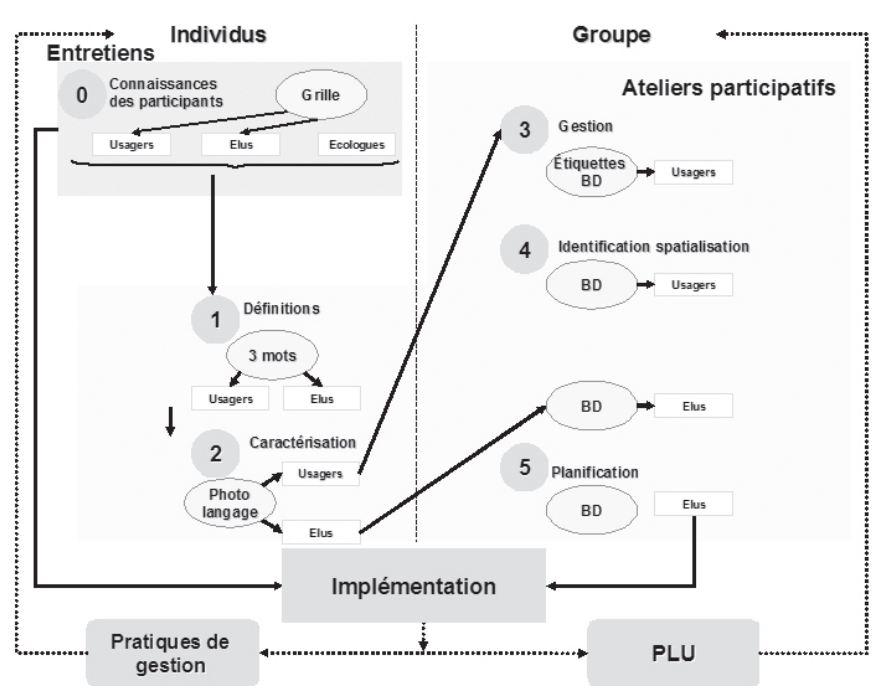

Étape 0 : étape préparatoire d'entretiens auprès de futurs participants (Girin, 1990) pour repérer les enjeux écologiques portés par les parties prenantes, identifier les représentations relatives aux objets de biodiversité, de TVB et collecter des photographies contextualisées.

Étape 1 : étape d'association continuée (Guimelli et Rouquette, 1992), dite des « 3 mots » pour faire énoncer les connaissances empiriques de chaque individu relatives aux objets de biodiversité, de TVB et réaliser l'installation de chaque participant (Girin, 1990) dans le groupe.

Étape 2 : étape de photolangage (Partoune, 2008) mobilisant des photographies contextualisées des objets et des menaces relatives aux objets de biodiversité, de TVB. Son objectif est l'explicitation orale des représentations de ces objets par chaque individu au sein du groupe.

Étape 3 : étape d'identification des menaces pesant sur les objets de biodiversité, les TVB mobilisant des logotypes pour chaque type de menace ainsi que des blocsdiagrammes (format $\mathrm{A} 3$ par binôme, puis format A0 pour le groupe). Elle a pour objectif de faire spatialiser les enjeux par binômes puis de réaliser une mise en commun au sein du groupe.

Étape 4 : étape d'identification des TVB mobilisant des blocs-diagrammes (format $\mathrm{A} 3$ par binôme, puis format $\mathrm{A} 0$ pour le groupe). Elle vise à faire spatialiser les lieux porteurs de biodiversité, les TVB et les éventuelles ruptures et menaces de continuités par binômes, puis à réaliser une mise en commun au sein du groupe.

Étape 5 : étape de prototypage des zonages PLU mobilisant des blocs-diagrammes pour traduire les représentations des TVB dans un langage réglementaire, par binômes, puis réaliser une mise en commun au sein du groupe (l'ordre de passage des binômes est laissé libre). 
Les résultats attendus du dispositif ALDOM sont triples. Ils consistent à :

1. Améliorer le niveau, la qualité, la circulation des informations et des connaissances requises par les acteurs en hybridant les régimes de productions des savoirs. Les acteurs apprennent les uns des autres. Cette hybridation des savoirs permet aux acteurs d'apprendre à décider et à agir en situation d'incomplétude des connaissances scientifiques, d'incertitude et de complexité des processus en jeu. En effet, les connaissances relatives au fonctionnement écologique des milieux à considérer dans le cadre des continuités écologiques n'est pas nécessairement stabilisé (van der Windt et al., 2008) et peuvent être d'autant plus perturbées que le nombre de parties prenantes intervenant dans leur gestion est démultiplié.

2. Confronter les valeurs et les représentations des parties prenantes. Il est possible de faire émerger les perceptions des acteurs, de cerner les valeurs dominantes et d'appréhender l'intelligibilité des objets écosystémiques qui constituent les TVB et la manière dont ils s'ancrent dans les lieux. ALDOM tend à faire converger ces représentations par des réflexions et des productions communes. Le résultat des interactions entre les participants débouche sur des productions collectives qui compensent, par une activité cognitive, les différences entre les représentations individuelles initiales (Vinck, 2009). En ce sens, ALDOM est un outil intermédiaire qui contribue à déplacer les points de vue des acteurs et participe « à la construction de compromis et de savoirs partagés » (Vinck, 2009, p. 59). Au final, les différences de perspectives entre les acteurs sont diluées, écrêtées par les transformations qui ont lieu entre les intentions individuelles et celles du groupe.

3. Partager la responsabilité dans la définition et la mise en œuvre des TVB. Les acteurs apprennent à définir ensemble le cadre de l'action et à l'expérimenter.

Ainsi, ALDOM constitue bien un dispositif d'apprentissage organisationnel et institutionnel qui met en relation "un ensemble résolument hétérogène comportant des discours, des institutions, [...], des décisions réglementaires, des lois, des mesures administratives, des énoncés scientifiques, des propositions philosophiques, morales, philanthropiques [...] du dit aussi bien que du non-dit » (Foucault, 1975, cité par Rey-Valette et al., 2010). Ces apprentissages organisationnels et institutionnels ont été identifiés et évalués lors d'une expérience concrète.

\subsection{Une application du dispositif ALDOM dans la commune de Morne-à-l'Eau en Guadeloupe}

Le dispositif participatif d'apprentissage ALDOM a été expérimenté en Guadeloupe en juillet 2013 dans la commune de Morne-à-l'Eau pour la définition des TVB dans une plaine dédiée à l'agriculture. La plaine de Grippon est un fossé d'effondrement entre deux failles tectoniques parallèles. Elle héberge plusieurs zones de captages d'eau dans la nappe phréatique qui se situe sous la 
commune, s'étend sur moins de $12 \mathrm{~km}$ le long d'un axe SE/NW avec une frange littorale à son extrémité NW et culmine dans sa partie amont à 24 m d'altitude. Elle mesure quelques $2 \mathrm{~km}$ dans sa partie la plus large. Dans sa partie amont, elle est mise en valeur par la culture de la canne à sucre. En aval, s'étend une zone de prairies comprenant majoritairement de l'élevage extensif mais aussi une agriculture vivrière (racines, tubercules) ou maraîchère. Cet espace prairial est également confronté à un phénomène de rurbanisation. Enfin, en interface avec la pleine mer, se trouvent le lagon du Grand Cul-de-Sac Marin et la zone de mangrove protégés par maints dispositifs (zones de cœur et de libre adhésion du Parc national de la Guadeloupe, propriétés du Conservatoire du littoral, zone RAMSAR, zones de réserve de biosphère, etc.). L'ensemble de la plaine est drainé par le canal des Rotours ( $6 \mathrm{~km}$ ) qui a été creusé au XIX ${ }^{\mathrm{e}}$ siècle (entre 1826 et 1830) pour permettre la culture de la canne à sucre. Aussi, les gestionnaires et usagers de la Plaine doivent raisonner sur un système écologique directement issu de l'ingénierie humaine et dont l'entretien et les dynamiques d'évolution sont fortement liés aux activités agricoles.

Les élus de la commune ont la charge de l'élaboration d'un document d'urbanisme (un PLU) qui intègre les TVB et de son application. Ce document doit être conçu de manière participative. Les travaux issus des ateliers ont pour objectif de servir la commune dans ce projet de révision. Ces ateliers ont rassemblé l'essentiel des parties prenantes devant contribuer à la conception et à la mise en œuvre des TVB : les élus de la commune (au nombre de 12) et les usagers dits « intermédiaires » de la Plaine (au nombre de 13). Ces acteurs sont qualifiés d'intermédiaires au sens où ils sont des responsables d'associations de mise en valeur du patrimoine, d'organisations professionnelles agricoles, de chasseurs. En cela, ils se trouvent dans l'appareil politique entre l'ensemble des citoyens pris individuellement et les élus.

Les résultats produits peuvent être synthétisés en trois points :

\section{Amélioration de la circulation des connaissances des acteurs et hy- bridation des régimes de productions des savoirs}

La démarche développée prépare plusieurs acteurs, dont les élus, à échanger, dialoguer, voire s'accorder en vue de la conception et de la mise en œuvre de TVB. Nos ateliers ont guidé les élus et les autres acteurs participants à réfléchir concrètement sur les types de zonages et les endroits pour lesquels la prise en compte des continuités écologiques et la préservation de la biodiversité constituent des enjeux majeurs pour la commune et sa périphérie (bloc diagramme focalisé sur la plaine de Grippon avec ouverture sur les franges alentours) ${ }^{4}$. La présence de chercheurs en sciences du vivant lors des ateliers a permis de vérifier

4 Ce bloc diagramme a été construit à partir de la lecture de paysages réalisée sur le terrain, l'interprétation de cartes (IGN - via Geoportail) et de photographies aériennes, l'intégration d'iconographies issues des documents du territoire (Atlas des paysages de l'Archipel Guadeloupe, Programme de protection, aménagement et mise en valeur du canal des Rotours et des canaux de la plaine de Grippon notamment), la contribution de chercheurs écologues pour la sélection de photos et d'information en lien avec les habitats. 
que les raisonnements tenus en matière de biodiversité sont cohérents avec les options de planification suggérées. La production des ateliers peut, de ce point de vue, pertinemment être intégrée dans un document de planification réel.

\section{Confrontation des valeurs et des représentations des parties prenantes}

La conduite simultanée de deux ateliers constitués chacun d'une catégorie d'acteurs (élus locaux, usagers et riverains de la plaine de Grippon) a permis de comparer et de confronter des visions produites par ces deux types de porteurs d'intérêts et d'enjeux distincts. La comparaison et la confrontation de ces visions portent un éclairage sur la possibilité ou non de trouver un compromis sur le territoire communal en matière d'action de préservation du vivant.

Le dispositif ALDOM a permis un processus de traduction/représentation/ médiation de la Plaine. Les résultats de ce processus mettent en exergue des convergences et des divergences entre le groupe des élus et celui des acteurs intermédiaires. Trois résultats convergents ressortent. Premièrement, les représentations relatives aux TVB sont identiques entre les deux catégories d'acteurs : les trames bleues reposent sur des linéaires déjà en place (ravines, canaux et mares) et les trames vertes sont conçues comme matricielles, c'est-àdire que c'est l'ensemble du territoire qui constitue la trame verte légitimant le fait que les espèces doivent y trouver les capacités de vivre, de se reproduire et de circuler. Second point de convergence, les résultats obtenus donnent à voir une faible capacité des parties prenantes à concevoir une forte écologisation des pratiques. En effet, les actions concrètes proposées par les participants à l'atelier pour une mise en œuvre effective des TVB consistent en des améliorations de l'existant. Il n'est jamais question de remettre en cause les paradigmes sous-jacents aux modes de productions (agricoles notamment) ainsi qu'aux processus de décision politique. Il n'y a pas de proposition d'action qui soit en rupture avec les décisions d'action en vigueur sur le territoire en matière de préservation de la biodiversité. Troisièmement, les ateliers se sont déroulés avec peu de controverses. Dans le groupe des élus, une unique controverse a porté sur la localisation des zones à bâtir. Dans le groupe des acteurs intermédiaires, une unique controverse a porté sur les causes de la raréfaction des oiseaux dans les espaces agricoles : le représentant des agriculteurs avançait l'argument qu'ils étaient trop chassés tandis que le représentant des chasseurs justifiait la concentration des oiseaux dans le bourg par des traitements phytosanitaires trop fréquents et polluants dans les espaces agricoles.

Les divergences entre groupes s'articulent autour des zones de discontinuités écologiques ainsi que des menaces qui entravent les continuités. Pour ce qui concerne les zones de discontinuités, les acteurs intermédiaires identifient le bourg et sa zone d'activités comme non perméables à la biodiversité et à la mobilité du vivant. Les élus conçoivent, en plus du bourg et de la zone d'activité au nord, la route qui rejoint un lieu-dit, bordé d'habitations entourées de jardins vivriers, comme source de discontinuité. Les divergences relatives aux menaces pour les discontinuités se fondent sur la nature de ces menaces. Les acteurs intermédiaires s'arrêtent sur les modes de production de certaines 
activités décrites comme polluantes (élevage et pratiques de garagistes notamment) alors que les élus discriminent la dynamique urbaine (au sein de leur commune comme dans la commune voisine) ainsi que la pratique répandue d'exploitation de carrières non déclarées et donc non autorisées.

\section{Partage de la responsabilité dans la définition et la mise en œuvre des TVB}

Le dispositif ALDOM a permis l'émergence de nouveaux rapports de prescriptions (Hatchuel, 2012) $)^{5}$ entre l'ensemble des participants. En effet, les élus et les forces vives de la commune ne peuvent ni dire qu'ils ne savent pas ce que sont, ni comment définir et mettre en œuvre, les TVB sur le territoire communal. Les acteurs intermédiaires savent que les élus sont capables de raisonner et définir les TVB de manière réfléchie. Les élus savent que les acteurs intermédiaires sont en mesure de raisonner, définir et prescrire des actions de gestion des TVB ; et chaque type de participant sait que l'autre type possède les mêmes perceptions (à quelques détails près eux aussi connus de tous). Finalement, chacun (élus, acteurs intermédiaires, observateurs) est tenu par un principe de responsabilité, personne ne peut prétexter un manque de connaissance, de savoir sur les enjeux de continuités écologiques pour justifier leur éventuelle non-prise en compte dans les documents de planification. Les parties prenantes ne peuvent non plus faire valoir que la biodiversité est affaire de spécialistes. Le groupe des élus a ainsi clairement pris conscience au cours du dispositif ALDOM que la conception du PLU de la commune était initiée et que celle-ci s'opérait pour le moment de manière discrétionnaire entre le maire et le cabinet d'urbanisme retenu.

Le dispositif ALDOM a permis d'accroître la dynamique collective intra et intergroupe favorisant une plus grande proximité de représentation entre les acteurs. Les apprentissages qui ont été rendus possibles par le dispositif ALDOM restent dans une boucle simple (Argyris et Schön, 1978) et ils concernent pour la plupart des aspects de coordination locale et de fonctionnement collectif. Sur le plan écologique, en revanche, aucun changement fondamental n'a été constaté quant aux représentations des acteurs par rapport aux entités vivantes non humaines. Ces résultats restent conformes à la dynamique générale observée sur le terrain.

\subsection{Discussion}

Dans cette section, nous nous interrogeons, dans une perspective réflexive, sur les résultats de l'expérience de gouvernance menée à Morne-à-l'Eau. Il s'agit pour nous de tirer parti de cette expérimentation d'action afin d'enrichir le processus de gouvernance des trames vertes et bleues tel qu'il a été mené

$5 \quad$ Selon Hachuel (2012) «certaines configurations de savoirs et de relations rendent possibles l' impact" d'une partie du savoir de A (noté $S A$ ) vers $B$. Nous dirons alors qu'il y a "rapport de prescription de $A$ vers $B$ pour $S A$ ". " (p. 54). 


\section{La gouvernance des trames vertes et bleues: de la nécessité d'une approche opérationnelle et réflexive}

en Guadeloupe. Nous discutons ainsi la contribution du dispositif ALDOM au cadre général de la GA.

\subsubsection{Une lecture dynamique de la démarche mise en œuvre}

Le dispositif ALDOM se situe avant, après, en même temps que d'autres dispositifs $^{6}$ qui permettent le « pilotage » du territoire concerné. Aussi, intègre-t-il d'autres cadres d'action qui, pris ensemble, constituent « un processus dynamique de coordination mobilisant des dispositifs multiples » (Rey-Valette et al., 2014). De cette manière, en élargissant le champ d'observation, on situe l'atelier participatif dans un ensemble de dispositifs qui constituent le processus de la gouvernance « en train de se faire » de l'aménagement et du développement de la commune de Morne-à-l'Eau.

L'analyse de l'ensemble de ces dispositifs montre une certaine stabilité des participants et des réseaux qui y sont impliqués. Les restitutions d'études et autres productions sont systématiques, elles constituent autant d'arènes et d'occasion d'exprimer un éventuel désaccord. Le partage de l'information par la présentation des productions de ces dispositifs est assuré ; il est même le moteur de l'animation du territoire. Pourtant, les controverses sont quasi inexistantes, qu'il s'agisse d'arènes fermées (auquel on ne participe que sur invitation) ou ouvertes (par exemple un débat public sur la place du bourg). Lorsqu'elles se manifestent, elles sont des joutes oratoires ou relatives à des détails de connaissance de la part de consultants et autres prestataires de service œuvrant sur la commune envers d'autres prestataires. Cela donne à voir un paysage politique et social relativement consensuel dans lequel les concurrences et les conflits se jouent entre prestataires éventuellement mais rarement (jamais) au sein de la communauté de Morne-à-l'Eau.

La commune acquiert, synthétise et articule une masse et une diversité de connaissances. Au final, on observe finalement que l'opérateur « savoir » ne cesse de croître alors que l'opérateur « relation » montre une certaine stabilité. Sur cette base, aucun changement de pratiques dans les modes de production visant à prendre en compte de nouveaux enjeux de biodiversité n'est ni pensé ni conçu. Et ce, malgré une capacité des participants à l'atelier à se représenter les écosystèmes de la commune comme étant continus, des chevelus (Latour, 1995). Si on s'en tient à faire de la TVB une opportunité de mettre en synergie, à l'échelle d'un territoire, des politiques publiques déjà existantes en matière de la biodiversité, le dispositif ALDOM tel qu'il s'est déroulé à Morne-à-l'Eau

6 Quelques mois auparavant, la commune avait initié le «programme de protection, d'aménagement et de mise en valeur du canal des Rotours et des canaux de la plaine de Grippon " à travers trois études (botanique, socio-culturelle et aménagement). Deux mois avant la mise en œuvre du dispositif ALDOM, se tenait le premier comité de pilotage du PLU de la commune. Dans le même temps, la collectivité démarre le dispositif relatif à l'Agenda 21 . L'ensemble de ces dispositifs sont eux-mêmes des instruments de mise en œuvre du programme électoral du maire élu en 2008 ; traduit par un primo dispositif, le " programme stratégique d'écocitoyenneté et d'écodéveloppement de Morne-à-1'Eau » au début de l'année 2011. 
apparait comme suffisant. Cependant, si l'on conçoit la conservation de la biodiversité dans une gamme plus large d'acceptions, une attention particulière doit être portée au caractère stratégique des agents impliqués dans le dispositif.

\subsubsection{Un processus d'amélioration continue}

Sur la base de notre expertise méthodologique, la région Guadeloupe nous a demandé de lui apporter notre concours pour l'élaboration de sa propre démarche de conception et de mise en œuvre de TVB et la mise en œuvre de son SRCE. Nous sommes restés impliqués sur ces deux terrains jusqu'en juillet 2015, ce qui nous a permis, à travers des entretiens qualitatifs, des participations à diverses réunions et comités, de collecter un matériau essentiel sur les enseignements à tirer de la démarche initiée avec le dispositif ALDOM expérimenté à Morne-à-l'Eau. Cela autorise un retour réflexif pour opérationnaliser la GA comme nous l'avons défini en section 1.

Nous mettons en avant que le principe d'un rééquilibre des participants parmi lesquels figureraient des acteurs stratégiques d'environnement (Mermet et al., 2005, Mermet, 2011) doit être observé. Ces derniers ont pour « mission principale de provoquer des changements appropriés » dans le système de gestion en place (Mermet et al., 2005, p. 130). Cette considération sur la dimension stratégique des acteurs est centrale, d'autant qu'en Guadeloupe, ces acteurs porteurs de vision différenciée existent.

Cette lecture réflexive et compréhensive nous a invités à raisonner et à intégrer cette dimension stratégique dans le cadre de la démarche de GA que nous avons initiée avec la région Guadeloupe pour la mise en œuvre des TVB à l'échelle de tout l'archipel. Sur cette base, nous avons organisé « un espace de travail ouvert » (Mermet et al., 2005) visant à rééquilibrer le débat entre agriculture et environnement en faveur d'enjeux de biodiversité. Il s'est agi de créer et de favoriser des situations qui ne sont pas exclusivement guidées par la coopération et la collaboration.

Cette approche critique nous amène à concevoir que la GA des TVB doit intégrer une dimension stratégique afin que leur gestion effective soit entreprise. Sur cette base, la GA constituerait un moyen de révéler les manières de concevoir un monde commun en vue de produire le changement effectif et serait en cela véritablement adaptative.

\section{Conclusion}

La conception et la mise en œuvre de politiques de conservation de la biodiversité que sont les trames vertes et bleues instaurent un contexte nouveau de complexité et d'incertitude qui appellent à la définition d'un cadre intégrateur conceptuel et méthodologique pour répondre aux enjeux de gouvernance soulevés dans ce contexte. Sur cette base, nous avons entrepris de revisiter le cadre de la GA en élaborant une proposition méthodologique qui conserve le 
primat d'une lecture compréhensive et réflexive légitimant la réalité empirique pour enrichir la théorie. L'expérience participative menée à l'échelle d'une commune de Guadeloupe nous a permis de souligner l'importance d'un cadre d'action tenant compte de la diversité des représentations et des intérêts des parties prenantes et qui opère les «traductions » nécessaires pour parvenir à créer un langage et une vision partagée des TVB et inventer les modalités de sa gestion qui impliquent une modification des normes d'action et des pratiques de gestion dans les espaces concernés.

À travers cet exemple, nous montrons que l'opérationnalisation de la GA des TVB doit passer par une approche stratégique de la gestion de l'environnement (Mermet et al., 2005 ; Mermet, 2011). Cette approche accorde une place centrale aux acteurs d'environnement qui ont pour mission de provoquer des changements pour une prise en compte réelle et intentionnelle des objets écologiques à conserver. Dans la perspective réflexive qui nous anime, ce résultat de l'expérimentation de l'action est intégré dans la démarche générale de la GA et sert de cadre pour la mettre en œuvre sur d'autres territoires et d'autres échelles.

\section{Bibliographie}

ALPHANDERY P. et FORTIER A. (2012), « La trame verte et bleue et ses réseaux : science, acteurs et territoires ", VertigO - la revue électronique en sciences de l'environnement [En ligne], Vol. 12, $\mathrm{n}^{\circ} 2$, septembre, mis en ligne le 20 septembre 2012 : http://vertigo.revues.org/12453 ; DOI : 10.4000/ vertigo.12453

ANGEON V. et CARON A. (2009), «Quel rôle joue la proximité dans l'émergence et la pérennité des modes de gestion durable des ressources naturelles? », Natures Sciences Sociétés, no 17, p. 361-372.

ARGYRIS C. et SCHÖN D. (1978), Apprentissage organisationnel : théorie, méthode, pratique, De Boeck (traduction française).

ANDERIES J.-M., JANSSEN M.-A. et OSTROM E. (2004), "A Framework to Analyze the Robustness of Social-Ecological Systems from an Institutional Perspective", Ecology and Society, Vol. 9, nº 1.

ANGEON V., CARON A., BIRARD C. et al. (2013), « Les apports de la gouvernance adaptative pour analyser les enjeux d'une mise en œuvre effective de la Trame Verte et Bleue. L'exemple du PNR des Volcans d'Auvergne ", Développement Durable des Territoires, Vol. 4, $\mathrm{n}^{\circ}$ 1, 17 p.

BERKES F. et FOLKE C. (1998), Linking social and ecological systems: management practices and social mechanisms for building resilience, Cambridge university press, New York.

BOUSQUET A. (2016), « Cartographie et participation », Carnets de géographes [En ligne], septembre 2016 : http://cdg.revues.org/625 
CASH D. W., ADGER W. N., BERKES F. et al. (2006), " Scale and cross-scale dynamics: governance and information in a multilevel world", Ecology and Society, Vol. 11, n 2, art. 8 : http://www.ecologyandsociety.org/vol11/iss2/ art8/

CHAFFIN B. C., GOSNELL H. et COSENS B. A. (2014), "A Decade of Adaptive Governance Scholarship: Synthesis andFuture Directions", Ecology and Society, Vol. 19, n 3, art. 56 : http://dx.doi.org/10.5751/ES-06824-190356

CHARVOLIN F., MATHEVET R. et VIMAL R. (2011), « La trame verte et bleue et son public », Quaderni, Vol. 76, p. 67-78.

CORDONNIER T. et GOSSELIN F. (2009), « La gestion forestière adaptative : intégrer l'acquisition de connaissances parmi les objectifs de gestion », Revue Forestière Française, n ${ }^{0}$ 61, p. 131-143.

COSENS B., GUNDERSON L., ALLEN C. et BENSON M. H. (2014), “Identifying Legal, Ecological and Governance Obstacles and Opportunities for Adapting to Climate Change", Sustainability, n ${ }^{\circ}$ 6, p. 2338-2356 :http://www.mdpi. com/2071-1050/6/4/2338 http://dx.doi.org/10.3390/su6042338

DIETZ T., OSTROM E. et STERN P. C. (2003), "The Struggle to Govern the Commons", Science, no 302, p. 1907-1912 : http://dx.doi.org/10.1126/ science.1091015

DUTRA L., THEBAUT O., BOSCHETTI F., SMITH A. et DICHMONT C. (2015), "Key Issues and Drivers Affecting Coastal and Marine Resource Decisions: Participatory Management Strategy Evaluation to Support Adaptive Management", Ocean \& Coastal Management, n 116, p. 382-395.

EBERHARD R., ROBINSON C., WATERHOUSE J. et al. (2009), "Adaptive Management for Water Quality Planning - from Theory to Practice", Marine and Freshwater Research, Vol. 60, p. 1189-1195.

FOLKE C., HAHN T., OLSSON P. et NORBERG J. (2005), "Adaptive governance of social-ecological systems", Annual review of environment and resources, Vol. 30, p. 441-473.

GIRIN J. (1990), «L'analyse empirique des situations de gestion : éléments de théorie et de méthode », in A. C. Martinet et Y. Pesqueux, Epistémologie des sciences de gestion, Economica, Paris, p. 141-182.

GUIMELLI C. et ROUQUETTE M.-L. (1992), « Contribution du modèle associatif des schèmes cognitifs de base à l'analyse structurale des représentations sociales », Bulletin de psychologie, Vol. 405, n 45, p. 196-202.

GUNDERSON L. (1999), "Resilience, flexibility and adaptive management - antidotes for spurious certitude?”, Ecology and Society, Vol. 3, n 1, 8 p.

HATCHUEL A. (2012), «Quel horizon pour les sciences de gestion? Vers une théorie de l'action collective ", in A. David, A. Hatchuel et R. Laufer, Les nouvelles fondations des sciences de gestion, Presse des Mines, Paris, p. 21-64. 
LASCOUMES P. et LE BOURHIS J.-P. (1998), « Le bien commun comme construit territorial », Politix, Vol. 11, n 42, p. 37-66.

LATOUR B. (1995), « Note sur certains objets chevelus », Nouvelle revue d'ethnopsychiatrie, $\mathrm{n}^{\circ} 27$, p. 21-36.

LEMOS M. C. et AGRAWAL A. (2006), "Environmental Governance”, Annual Review of Environment and Resources, Vol. 31, p. 297-325 : http://dx.doi. org/10.1146/annurev.energy.31.042605.135621

MERMET L. (2011), "Strategic Environmental Management Analysis: Adressing the Blind Spots of Collaborative Approaches", Idées pour le débat, IDDRI - SciencePo, n 5 , p. 1-34.

MERMET L., BILLE R., LEROY M., NARCY J.-B. et POUX X. (2005), « L'analyse stratégique de la gestion environnementale : un cadre théorique pour penser l'efficacité en matière d'environnement », Natures Sciences Sociétés, n 13, p. 127-137.

OLSSON P., GUNDERSON L. H., CARPENTER S. R. et al. (2006), "Shooting the rapids: navigating transitions to adaptive governance of social-ecological systems", Ecology and Society, Vol. 11, n 1, 21 p.

PAHL-WOSTL C. (2009), "A conceptual framework for analysing adaptive capacity and multi-level learning processes in resource governance regimes", Global Environment Change, n ${ }^{\circ} 19$, p. 354-365.

PAHL-WOSTL C., CRAPS M., DEWULF A. et al. (2007), "Social Learning and Water Resources Management", Ecology and Society, Vol. 12, n 2, 22 p.

PARTOUNE C. (2008), « Le photolangage », Hyperpaysage, Laboratoire de méthodologie de la géographie de l'Université de Liège et de l'Institut d'éco-pédagogie : http://www.hyperpaysages.be/spip/spip.php?article37

PLANCHAT-HERY C., LARADE A. et ANGEON V. (2013), Restitution d'une démarche de concertation sur la biodiversité et la trame verte et bleue. Commune de Morne-à-l'Eau en Guadeloupe, 43 p.

PLANCHAT-HERY C. (2011), Du paysage aux intentions d'aménagement : usage des représentations paysagères pour la planification de l'agriculture dans les territoires péri-urbains, Thèse de doctorat, Université Blaise Pascal - Centre d'Études et de Recherches Appliquées au Massif Central, à la Moyenne Montagne et aux Espaces Fragiles (CERAMAC), 305 p.

REY-VALETTE H., CHIA E., MATHE S. et al. (2014), « Comment analyser la gouvernance territoriale? Mise à l'épreuve d'une grille de lecture », Géographie, Économie, Société, Vol. 16, nº 1, p. 65-89.

REY-VALETTE H., CHIA E., SOULARD C. et al. (2010), «Innovations et gouvernance territoriale : une analyse par les dispositifs », Innovation and Sustainable Development in Agriculture and Food, ISDA 2010 Montpellier, 10 p. 
SAINSBURY K. J., PUNT A. E. et SMITH A. D. M. (2000), “Design of Operational Management Strategies for Achieving Fishery Ecosystem Objectives”, ICES Journal of Marine Science, $\mathrm{n}^{\circ}$ 57, p. 731-741.

SMITH A.D.M., SAINSBURY K.J. et STEVENS R.A. (1999), “Implementing Effective Fisheries- Management Systems - Management Strategy Evaluation and the Australian Partnership Approach", ICES Journal of Marine Science, No. 56, p. 967-979.

TERMEER C. J. A. M., DEWULF A. et VAN LIESHOUT M. (2010), “Disentangling Scale Approaches in Governance Research: Comparing Monocentric, Multilevel, and Adaptive Governance", Ecology and Society, Vol. 15, No. 4, 15 p.

THEAU J.-P., CHABALIER C., PIQUET M., CAYRE P., DELMAS D., VIOLEAU S. et FARRUGIA A. (2012), "Construire des outils en partenariat entre recherche et développement. LE diagnostic des pratiques fourragères en zone fromagère AOP du Massif Central », Fourrages, n 209, p. 69-78.

VAN DER WINDT H. J. et SWART J. A. A. (2008), "Ecological Corridors, Connecting Science and Politics: The Case of the Green River in Netherlands", Journal of applied ecology, No. 45, p. 124-132.

VIMAL R., MATHEVET R. et MICHEL L. (2012), « Expertise et concertation : peut-on penser collectivement la protection intégrée de la nature? », Natures, Sciences, Sociétés, No. 20, p. 415-424.

VIMAL R., MATHEVET R. et THOMPSON J. D. (2012), "The Changing Landscape of Ecological Networks", Journal for Nature Conservation, No. 20, p. $49-55$.

VIMAL R. et MATHEVET R. (2011), « La carte et le territoire : le réseau écologique à l'épreuve de l'assemblée cartographique », Cybergeo : European Journal of Geography [En ligne], Environnement, Nature, Paysage, document 572, URL : http://cybergeo.revues.org/24841.

VINCK D. (2009), « De l'objet intermédiaire à l'objet-frontière, vers la prise en compte du travail d'équipement », Revue d'Anthropologie des Connaissances, Vol. 3, No. 1, p. 51-72.

WALKER B., HOLLiNG C. S., CARPENTER S. R. et KINZIG A. (2004), "Resilience, Adaptability and Transformability in Social-Ecological Systems", Ecology and Society, Vol. 9, No. 2, art. 5 : http://www.ecologyandsociety.org/ vol9/iss2/art5 